\title{
Durable complete remission of poor performance status metastatic lung adenocarcinoma patient treated with second-line erlotinib: a case report
}

This article was published in the following Dove Press journal:

OncoTargets and Therapy

6 September 2017

Number of times this article has been viewed

\author{
Dragana Jovanovic ${ }^{1,2}$ \\ Ruza Stevic ${ }^{1,2}$ \\ Marta Velinovic ${ }^{2}$ \\ Milica Kontic ${ }^{1,2}$ \\ Dragana Maric ${ }^{1,2}$ \\ Jelena Spasic ${ }^{3}$ \\ Davorin Radosavljevic ${ }^{3}$ \\ 'Faculty of Medicine, University \\ of Belgrade, Belgrade, Serbia; \\ 'University Hospital of Pulmonology, \\ Clinical Center of Serbia, Belgrade, \\ Serbia; ${ }^{3}$ Institute for Oncology and \\ Radiology of Serbia, Belgrade, Serbia
}

Correspondence: Milica Kontic

University Hospital of Pulmonology,

Clinical Center of Serbia, Belgrade, Koste

Todorovica 26, Belgrade 11000 , Serbia

Tel +38I 63554963

Email milicakontic@yahoo.com
Abstract: This paper presents a rare case of an elderly patient treated with erlotinib for disseminated lung adenocarcinoma with poor performance status (Eastern Cooperative Oncology Group performance status [PS]3). This treatment led to a long duration of complete remission according to Response Evaluation Criteria in Solid Tumors 1.1 - almost 7 years (81 months) of progression-free survival (PFS) and overall survival (OS) of 10 years by March 2017. The treatment with erlotinib started in September 2008 and it was well tolerated with no adverse effects. Mutation analyses (real-time polymerase chain reaction method) revealed deletion of EGFR (epidermal growth factor receptor) gene and wild-type Kirsten-ras protein gene in exon 19. In May 2015, the patient relapsed with jaundice and enlarged lymph nodes of the liver hilum, with no other metastasis, PS 2. Biopsy confirmed metastasis of lung adenocarcinoma. EGFR molecular testing did not reveal T790M mutation. Treatment was continued with gemcitabinecisplatin chemotherapy. A total of six cycles were administered with nearly complete response and Eastern Cooperative Oncology Group performance status 0. Further on, gemcitabine monotherapy has been administered with nearly complete response maintained and OS of 10 years by March 2017. This report describes an extremely rare case of a poor performance patient with advanced metastatic adenocarcinoma harboring EGFR mutation - deletion in exon 19 - who was receiving salvage erlotinib and had a complete response with 81 months of PFS followed by a relapse and subsequent chemotherapy which led to nearly complete response, with an OS of 10 years by March 2017. Such a complete response to tyrosine kinase inhibitor therapy in a poor PS patient, with long PFS and OS achieved, justifies tyrosine kinase inhibitor treatment approach in poor PS patients with EGFR-sensitizing tumors, and furthermore points to the feasibility of administering chemotherapy at the time of relapse.

Keywords: lung adenocarcinoma, erlotinib, exon 19 deletion, EGFR, durable remission

\section{Introduction}

The detection of epidermal growth factor receptor (EGFR) mutations in tumor tissues has been used to predict the response of tyrosine kinase inhibitor (TKI) treatment and thereby guide the treatment for advanced non-small cell lung cancer (NSCLC).

Although the EGFR TKIs - gefitinib, erlotinib, and afatinib - have changed the treatment paradigm for NSCLC among those with EGFR mutation-positive disease, most patients experience disease progression after $\sim 12$ months of treatment (range, 8-14 months) due to mutations causing secondary resistance. ${ }^{2,3}$ Progression of EGFRdriven NSCLC after EGFR-TKI therapy presents a significant challenge for clinicians with more than half of cases of progression attributed to acquired resistance due to the T790M mutation. In $18 \%$ to $30 \%$ of patients, the cause of resistance is unknown., ${ }^{4,5}$ 
There are different options for treatment of progressive disease in EGFR mutation-positive metastatic NSCLC. Because systemic progression after EGFR-TKI is thought to result from the emergence of EGFR-TKI-resistant clones, which may be confirmed with molecular testing at progression, switching to chemotherapy after progression is common. ${ }^{5}$ An effective treatment option for patients with metastatic EGFR T790M mutation-positive NSCLC who progressed on or after EGFR-TKI therapy is osimertinib, a kinase inhibitor that targets this most prevalent secondary mutation.

This report describes an extremely rare case of a poor performance patient with advanced metastatic adenocarcinoma harboring EGFR mutation - deletion in exon 19 who was receiving salvage erlotinib and had a complete response with 81-month-long progression-free survival (PFS).

Erlotinib treatment was tolerated well, without any adverse events (AEs). This was followed by a relapse and subsequent chemotherapy, which led to a nearly complete response, with an overall survival (OS) of 10 years by March 2017. Written informed consent has been provided by the patient to have the case details and all accompanying images published.

This case confirms that EGFR-sensitizing mutation tumors have beneficial predictive and prognostic significance and justifies treatment with TKIs in poor performance status patients with tumors harboring EGFR-sensitizing mutations.

\section{Case report}

A 59-year-old, non-smoking, Caucasian female presented in February 2007 with a CT scan of the thorax revealing a tumor mass of $52 \times 49 \mathrm{~mm}$ in the upper lobe of the left lung with a few enlarged ipsilateral mediastinal lymph nodes, up to $18 \mathrm{~mm}$. She had no concomitant diseases. Bronchoscopy with transbronchial needle aspiration confirmed adenocarcinoma with negative mediastinal lymph node biopsy findings. Following bone scintigraphy and ultrasonography of the abdomen with normal finding, left upper lobe lung resection with mediastinal lymphadenectomy was performed in February 2007. Adenocarcinoma, acinopapillary subtype, g2, pT2N1Mo was verified (Figure 1A). Two cycles of adjuvant carboplatin/gemcitabine were given, but the therapy was
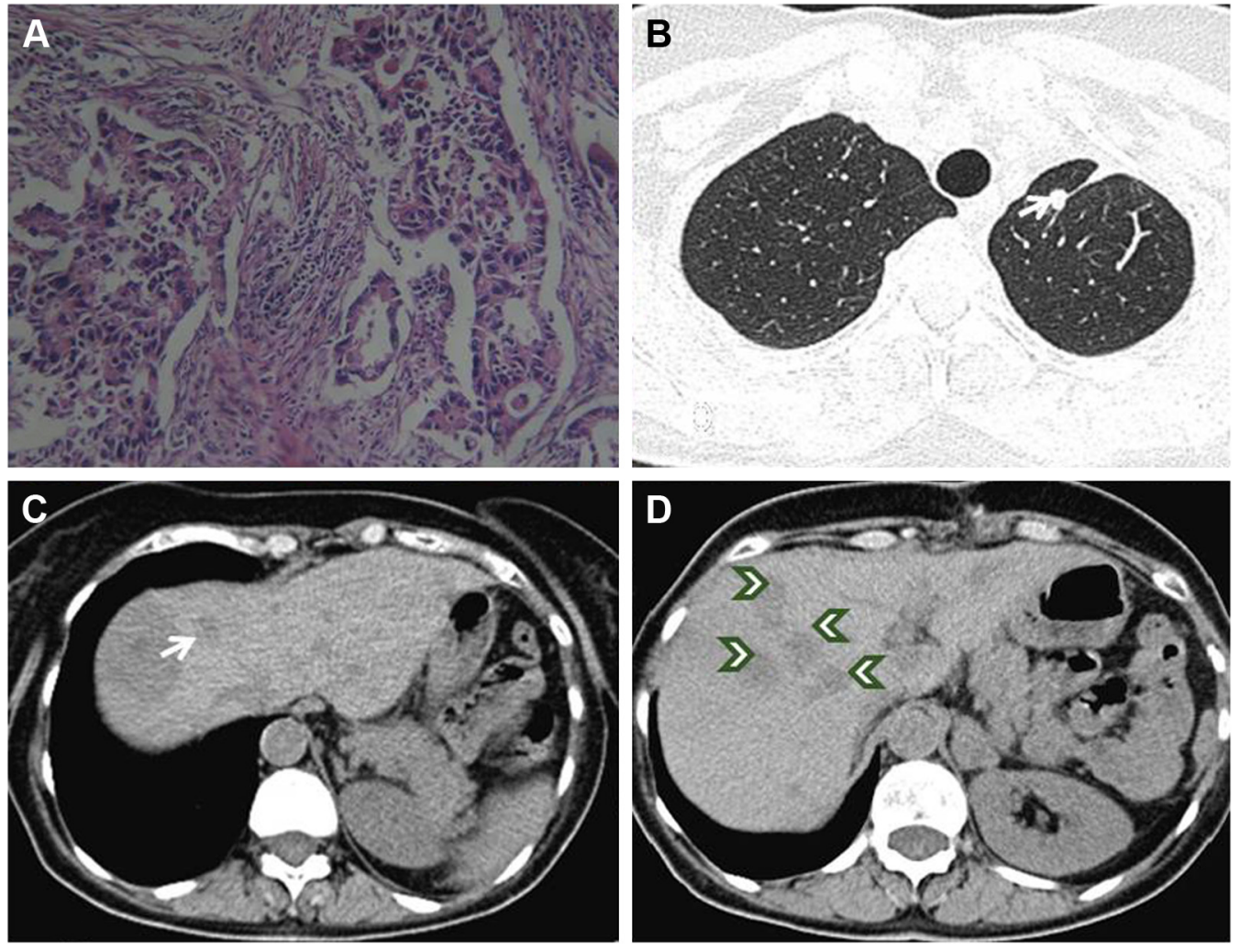

Figure I Initial histology and CT findings.

Notes: (A) Microphotograph of adenocarcinoma, acinopapillary subtype. H\&E staining, magnification $\times 40$. (B) Axial CT in lung window with solitary nodule in left upper lobe (arrow). (C) Axial CT of abdomen shows solitary liver metastasis (arrow) close to ablation zone in eighth segment. (D) Follow-up CT revealed multiple liver metastases (arrowheads). 
stopped due to serious AEs, persistent vomiting and obstipation with the development of ileus. There was no sign of relapse and the patient remained in an excellent condition (Eastern Cooperative Oncology Group Performance Status [ECOG PS] 0) until the end of 2007. A year after operation, in February 2008, the disease relapsed and radiofrequency ablation (RFA) of solitary liver metastasis was performed. Three months later, in May 2008, multi-slice computed tomography (MSCT) scan of the thorax and abdomen revealed one metastasis in the left lung $(8 \mathrm{~mm})$ and one in the liver $(11 \mathrm{~mm})$ in the eighth segment (Figure $1 \mathrm{~B}$ and $\mathrm{C}$ ) and suspected metastases in enlarged adrenal glands (the lesion in left $32 \mathrm{~mm}$ and in right $14 \mathrm{~mm}$ in diameter). Following surgeon's decision, explorative laparoscopy was performed with the intent of excision of the new solitary liver lesion, but at the operation 13 liver metastases up to $1 \mathrm{~cm}$ in size were identified, with metastases in both adrenal glands. After two cisplatin/docetaxel cycles, further disease progression with PS deteriorated (PS 3) was observed. The patient was advised to pursue the EGFR mutation analysis of the tumor elsewhere, since, at that time, there was no technical possibility to perform this test in our country. In September 2008, a control CT scan of the thorax and abdomen registered further disease progression and the enlargement of liver metastases (Figure 1D). Then, by the end of September 2008, erlotinib $150 \mathrm{mg}$ per day as salvage second-line treatment was introduced. This was the only TKI available in the country at that time. A dramatic improvement of PS was noted after 2 weeks, with ECOG PS changing to zero and control CT scan showing a good initial response some degree of tumor regression. In December 2008, MSCT of the thorax and abdomen verified excellent tumor response after 2 months of treatment with significant regression of liver metastases - from 13 down to only 2 (Figure 2A) and a complete regression of lung and adrenal metastases. Complete regression according to the RECIST 1.1 (Response Evaluation Criteria in Solid Tumors 1.1) continued further. MSCT scan in March 2009 showed no metastasis (Figure 2B and C). Each consecutive evaluation, MSCT scans from January 2010 to February 2015, showed the maintenance of the complete response (Figure 2D-F).

Mutation testing in January 2012 by real-time PCR (Scorpions-ARMS) on paraffin-embedded tumor blocks (surgical piece) detected deletion of Exon 19 and wild-type KRAS (Kirsten-ras protein gene).

The patient was on Erlotinib therapy alone (with no concurrent medication) for 81 months with no AEs. During this time, the ECOG PS remained 0 and the CT scans of the thorax confirmed durable complete response (last performed in February 2015).

In May 2015, the patient relapsed with jaundice as the first symptom. Positron emission tomography/computed tomography (PET/CT) showed increased fluorodeoxyglucose uptake in lymph nodes of the liver hilum, about $5 \mathrm{~cm}$ in diameter, with no other pathologic findings (Figure 3A). In June 2015, surgical biopsy was performed, which confirmed metastasis of lung adenocarcinoma. EGFR molecular testing did not reveal T790M mutation.
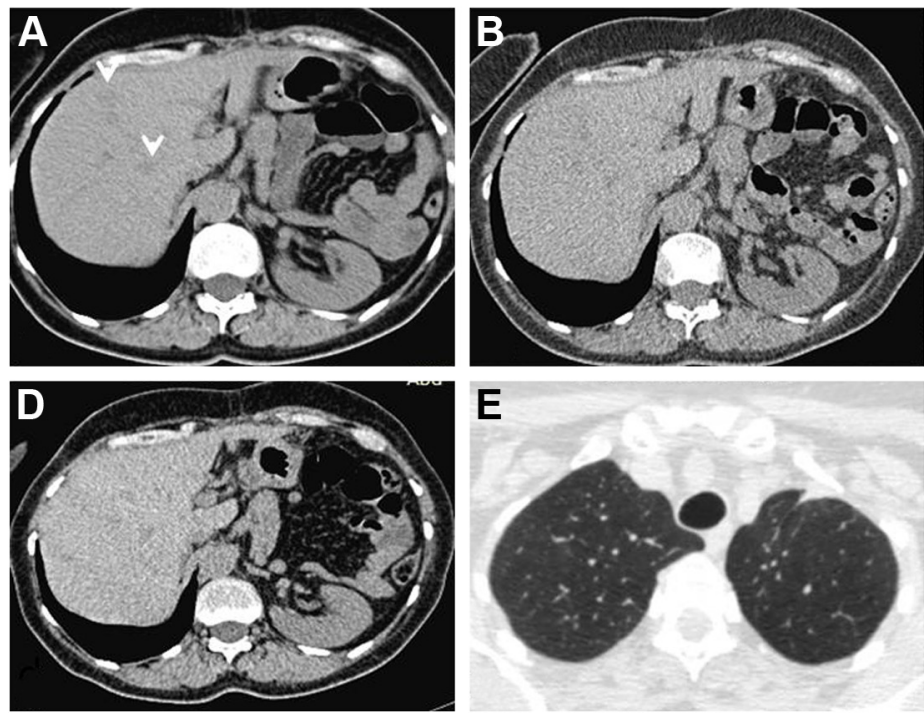
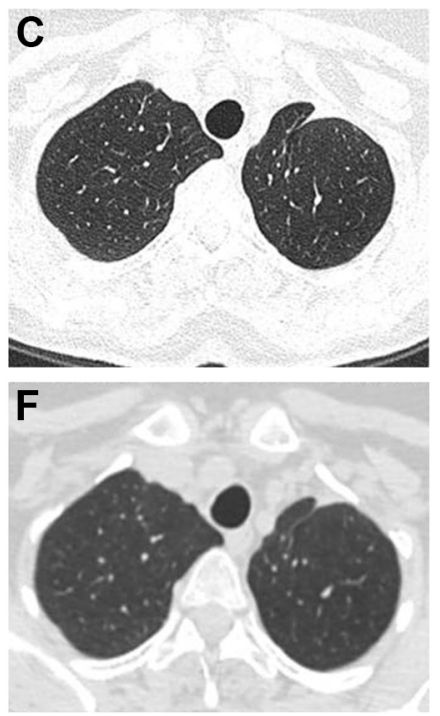

Figure 2 Subsequent CT findings.

Notes: (A) Follow-up CT after 2 months' treatment shows only two small liver metastases (arrowheads). (B and C) Follow-up CTs of abdomen and thorax from March 2009 show no metastases. (D) Follow-up CT of abdomen from 2012 without liver metastases. (E and F) Follow-up CTs of thorax from 2010 and 2012 show no lung metastases. 

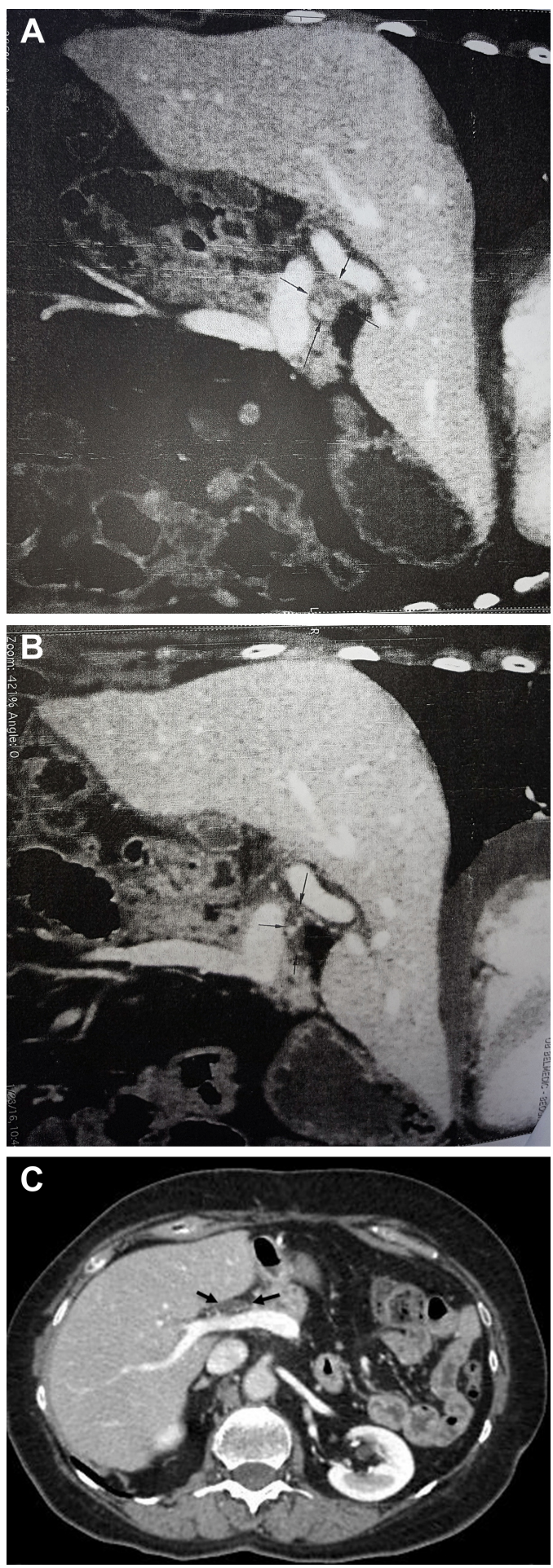

Figure 3 CT finding of disease progression on erlotinib treatment. Notes: (A) Enlarged lymph nodes of the liver hilum - about $5 \mathrm{~cm}$ in diameter (arrows). (B) Follow-up after six cycles of gemcitabine-cisplatin chemotherapy; nearly complete response (arrows). (C) Contrast-enhanced axial CT of abdomen shows small hypodenze node in the liver hilim is unchanged compared with previous CT; nearly complete response (arrows).

The treatment was continued with gemcitabine-cisplatin chemotherapy. A total of six cycles were administered by February 2016 with nearly complete response according to the RECIST 1.1 (Figure 3B) and ECOG PS of 0. Further on, gemcitabine monotherapy has been administered, with no AEs, which maintained nearly complete response (Figure 3C). By March 2017, an OS of 10 years has been reached.

\section{Discussion}

EGFR TKIs have revolutionized the treatment for patients with advanced NSCLC and EGFR-activating mutations.

The mutation L858R in exon 21 and the deletion in exon 19 account for $>85 \%$ of all clinically important mutations related to TKI sensitivity. ${ }^{1,3}$ The female gender, adenocarcinoma histology, never-smoking status, and Asian ethnicity are associated with a significantly greater likelihood of tumors harboring EGFR-activating mutations and thus being responsive to TKI therapy. In Caucasians, only about $11 \%$ of patients (range $10 \%-15 \%$ ) with NSCLC have tumors that harbor EGFR-activating mutations. ${ }^{6,7}$

Three oral EGFR TKIs - gefitinib, erlotinib, and afatinib are currently recommended as first-line therapy in patients with advanced or metastatic NSCLC harboring EGFRactivating mutations, based on the success of clinical trials in EGFR mutation-positive selected populations. ${ }^{2,8-13}$

According to the Surveillance, Epidemiology, and End Results (SEER) program data, the 5-year relative survival for unselected patients with distant-stage NSCLC is $4.5 \% .^{14}$ Likewise, prior lung cancer studies have provided similar estimates of 5 -year relative survival $<5 \% .{ }^{15,16}$ However, gefitinib and erlotinib used in clinical trials as a first-line treatment for patients with advanced NSCLC harboring activating EGFR mutations led to response rates (RRs) of $56 \%-74 \%$, with a median PFS of $10-14$ months and an OS of 20-30 months. ${ }^{2,8,10,17}$

Although generally acknowledged that TKIs have transformed the outcome of some patients with NSCLC, there has been scarce data to demonstrate the impact of EGFRTKIs on the long-term survival of patients with activating mutations treated with these agents until recently. The trials LUX-Lung 3 and LUX-Lung 6, which studied OS advantage, showed that median survival on TKIs was prolonged by 12-13 months compared to chemotherapy for patients with exon 19 deletions (but not for the entire population in the trials). ${ }^{11,12,18-21}$ The lack of OS advantage in clinical trials might be attributable to high crossover rates from chemotherapy to EGFR-TKIs. ${ }^{20,21}$

However, outcomes in patients treated with EGFR-TKIs vary widely, suggesting heterogeneity in the underlying clinical or genetic factors, which may further modify patient's response and influence the ultimate course of disease. ${ }^{5,13,17}$

Our patient had all favorable clinical characteristics (female gender, a negative smoking history, and adenocarcinoma 
histology) and, consistent with it, EGFR-activating mutation - deletion in exon 19.

In a retrospective study, Shigematsu and Gazdar reported the genomic analysis of more than 2000 NSCLCs; EGFR mutations were found to be more common in adenocarcinoma $(30 \%)$ and more frequent in lung cancer in never-smokers (45\%) than ever-smokers (7\%). Many published reports and studies noted similar overall RRs and OS rates despite different geographical locations and regardless of which EGFRTKI was used. ${ }^{22,23}$ The RRs generally ranged from $62 \%$ to $83 \%$, and OS ranged from 23 to 39 months. ${ }^{2,8-12,17}$ However, there are marked differences in response to TKIs' treatment, depending on the type of the activating EGFR mutation.

At present, there is a clear evidence that the presence of EGFR mutations has a predictive value as well as a prognostic one. $2,3,9,10,13,17,24,25$

There are numerous reports on response to TKIs, including erlotinib therapy. The response can be prompt, even in diffuse brain metastatic disease, occasionally leading to a remarkable survival and PFS periods. ${ }^{26-30}$

Riely et al found $24 \%$ of EGRF mutations prevalence with the predominance of EGFR exon 19 deletion (61\%), and the patients with this predominant type of mutation had significantly longer median survival than patients with EGFR L858R. ${ }^{30}$

Spanish Lung Cancer Group (SLCG) analyzing more than 2000 NSCLC cases noted EGFR mutations positive in $16.6 \%$, EGFR exon 19 deletion detected in $62.2 \%$, and the L858R point mutation in $37.8 \%$ of the cases. ${ }^{10,31}$ The RR was $70.6 \%$ with $12.2 \%$ complete responses, and a better outcome was associated with the exon 19 deletion than with the L858R mutation.

Gazdar et $\mathrm{al}^{1}$ came to a similar conclusion: RR to TKIs in patients with EGFR exon 19 deletion was $70 \%-90 \%$, and that in patients with EGFR exon 21 mutation was 20\%-67\%. Patients with EGFR exon 19 deletion also had a significantly longer median survival compared to those with L858R mutation (26-34 vs 8-17 months, respectively).

Our case is the very example of this superior outcomedurable complete response associated with the exon 19 deletion. $^{1}$

In a recently published retrospective study of 5-year survival among 137 patients with EGFR-mutant metastatic lung adenocarcinoma treated with an EGFR-TKI, the median PFS was 12.1 months (95\% CI: 10.2-13.5) and OS was 30.9 months (95\% CI: 28.2-35.7), while 20 patients (14.6\%) survived for at least 5 years (range 5.0-7.7 years). ${ }^{17}$ The presence of an EGFR mutation in exon 19 versus in exon 18 or 21 was associated with longer survival (hazard ratio $=0.55,95 \%$ confidence interval: $0.38-0.79, p=0.001$ ). No difference in survival was seen among patients who received EGFR-TKI treatment as first-, second-, or third-, or greater-line therapy (median OS 30.9, 30.9, and 37.2 months, respectively; $p=0.74)$. The association between exon 19 deletion and prolonged survival in patients with advanced NSCLC treated with EGFR-TKIs has been reported by several groups. ${ }^{11,12,21,30,31}$ However, the biological mechanisms underlying these apparent differences in outcome, rendered by the EGFR mutations, remain largely unknown.

A long-duration complete response in poor performance status patients, such as our patient, is extremely rarely observed..$^{32}$ In addition, significant treatment-related side effects in this subset of patients are often observed. ${ }^{33}$

The advantage of oral administration of EGFR-TKIs along with their favorable toxicity profile led to a considerable interest in evaluating their efficacy in poor PS patients with NSCLC. Such was our patient when the treatment with erlotinib was initiated and it subsequently lasted for 81 months, with no adverse effects.

Patients with PS 2 have traditionally been excluded from clinical trials and from receiving standard platinum-based chemotherapy because of their expected high rate of toxicity after such therapy.

In the SLCG study (217 EGFR mutation-positive lung cancer patients), there were no significant differences in PFS related to performance status. ${ }^{10,31}$

Lin and Yang summarized the results of erlotinib or gefitinib in the subsets of patients with advanced NSCLC in poor performance status. ${ }^{34,35}$ Several Phase II trials had underrepresented patient population with ECOG PS of 2, while those of PS of 3 or 4 were not included in all chemotherapy treatment studies. Only few studies were designed specifically to establish standard treatment for poor PS patients.

In the randomized Phase II trial of erlotinib versus erlotinib plus carboplatin/paclitaxel in patients with advanced NSCLC and impaired performance status (SWOG S0709), $6.2 \%$ of patients in the erlotinib group and $19.0 \%$ in combination group experienced serious treatment-related grade 4 AEs. There were no treatment-related deaths. This study has shown the feasibility of using a combination therapy, erlotinib plus chemotherapy, in poor performance status patients. ${ }^{36}$

Results of relevant clinical trials have not shown significant difference regarding OS and PFS in poor PS patients versus good PS patients treated with TKIs; rather, they suggest that the concern was the safety issue in the population of poor PS patients. $2,9,10,21,25$

Erlotinib was shown to have comparable safety profile with gefitinib and treatment-emergent AEs were noted 
in $>10 \%$ in either group, with no significant differences between the two TKIs, erlotinib and gefitinib. ${ }^{37}$

A pooled safety analysis of the incidence of severe (grade $\geq 3$ ) toxicity according to the type of EGFR-TKI administered was done using data extracted from prospective clinical trials including advanced NSCLC patients harboring $E G F R$ mutations. There were clinically significant differences in the occurrence of hepatotoxicity, rash, and diarrhea among patients treated with these three EGFR-TKIs, although such differences had been directly investigated in only a limited number of patients to date. ${ }^{25}$

Although there are some published case reports on the remarkable response to TKI treatment in poor performance status patients with advanced NSCLC, long-duration complete response in poor performance status patients, as is the case described in this paper, is extremely rarely noted. Durable, complete pathologic responses of 10 and 30 months have been described in two patients with metastatic adenocarcinoma of the lung with multiple bone metastases treated with erlotinib. ${ }^{32}$

More often, long-term partial remission achieved with erlotinib therapy is reported. ${ }^{8-12,21,35}$

Our patient has had disease relapse after 81 months of complete response (PFS) at 67 years of age. Jaundice was a first symptom. Metastasis was in lymph nodes of the liver hilum. Patient had deterioration of PS (ECOG PS 2). Subsequent surgical biopsy was performed and metastasis of lung adenocarcinoma was confirmed. EGFR molecular testing did not reveal T790M mutation. The treatment choice was chemotherapy, cisplatin/gemcitabine regimen. A total of six cycles were administered with nearly complete response and ECOG performance status 0. Further on, gemcitabine monotherapy has been administered with nearly complete response maintained and survival time of 10 years by March 2017.

Most patients with EGFR mutation-positive disease experience disease progression after $\sim 12$ months (range 8-14 months) of treatment due to secondary resistance mutations, and this progression of EGFR-driven NSCLC after EGFR-TKI therapy presents a significant challenge for clinicians..$^{2-5,13}$ There are several mechanisms of acquired resistance to EGFR-TKIs, the most common being (in $\sim 60 \%$ of cases) the result of a secondary point mutation in exon 20 of the EGFR gene, T790M [15-20]. In 18\%-30\% of patients, the cause of resistance is unknown. ${ }^{4,5}$

Few effective options were available on progression until the introduction of osimertinib, a kinase inhibitor that targets the most prevalent T790M mutation, recently approved for treatment of patients with metastatic EGFR
T790M mutation-positive NSCLC, who progressed on or after EGFR-TKI therapy. Thus, performing molecular testing at progression is critically important to identify patients with or without the T790M mutation. Furthermore, plasma-based testing for the T790M mutation is another option that may prevent the need for a metastasis biopsy in a significant subset of patients. Treatment options are based on the mechanism of resistance to the initial EGFR-TKI treatment, whether the patient is symptomatic or asymptomatic, which is the metastatic site, and whether isolated or multiple lesions. Because systemic progression after EGFR-TKI is thought to result from the emergence of EGFR-TKI-resistant clones, switching to chemotherapy after progression is common, although it is justified in case of exclusion of T790M as the resistance mechanism as was the case with our patient who is alive and well with nearly complete remission 10 years after diagnosis of lung adenocarcinoma was established, and is aged 69 now. ${ }^{13}$

Although many studies have focused on first-line EGFRTKI treatment for elderly patients, the treatment efficacy of second-line salvage chemotherapy for elderly patients remains unknown with the controversy over which chemotherapy regimen or agent to use after disease progression due to acquired resistance after first-line EGFR-TKI treatment. A retrospective analysis was performed on 330 stage IV adenocarcinoma patients with an EGFR mutation who received first-line TKI treatment and, following disease progression, subsequent salvage chemotherapy, 160 being $\geq 70$ years old (elderly group) and 170 being $<70$ years old (younger group). The RR and PFS with first-line TKI treatment were not significantly different. The elderly group had shorter median survival. The RRs to salvage chemotherapy were not significantly different regarding the age group. PFS did not differ significantly as well between the younger and older patients (5.1 vs 3.5 months, $p=0.070$ ). However, the OS of the younger patients was significantly longer than that of the older group ( 26.5 vs 21.3 months, $p=0.021$; and 18.2 vs 10.9 months, $p=0.014$, respectively, for EGFR-TKI and subsequent salvage chemotherapy). Elderly patients who received second-line salvage chemotherapy had a median OS of $\sim 10.9$ months, which is longer than the OS of patients who received the best supportive care. ${ }^{5}$

Goldberg et al compared chemotherapy plus erlotinib with chemotherapy alone in advanced NSCLC patients who had received first-line EGFR-TKI and had acquired resistance. An improved RR (63\% vs 21\%; $p=0.02$ ) was seen, but no significant difference was found in PFS (4.4 vs 4.2 months; $p=0.5$ ) or OS (14.2 vs 15.0 months; $p=0.37) .{ }^{38}$ 


\section{Conclusion}

Individualized lung cancer treatment in the subgroup of patients with sensitizing EGFR mutation has brought about a benefit in survival and quality of life in this subgroup of patients, which has not been obtained until now with modern chemotherapy. We presented the case of a poor performance status patient (PS 3) with metastatic lung adenocarcinoma harboring deletion in exon 19 of EGFR gene, treated with erlotinib without any AE, who achieved a durable complete response of over 81 months with excellent quality of life. Following histologic confirmation of tumor relapse, T790 mutation negative, salvage chemotherapy cisplatin/ gemcitabine was introduced with nearly complete response after six cycles, continued with monotherapy gemcitabine, well tolerated. The patient is alive 10 years after lung adenocarcinoma was diagnosed, maintaining nearly complete response. Such rare case of complete response to TKI therapy in a poor PS patient, with long PFS and OS achieved, justifies TKI treatment approach in poor PS patients with EGFRsensitizing tumors and furthermore points to the feasibility of administering chemotherapy at the time of relapse.

\section{Acknowledgment}

This work was supported by the Ministry of Education and Science of Serbia, contract number 175,046, 2011-2014 and III 43,007.

\section{Disclosure}

The authors report no conflicts of interest in this work.

\section{References}

1. Gazdar AF. Activating and resistance mutations of EGFR in non-small cell lung cancer: role in clinical response to EGFR tyrosine kinase inhibitors. Oncogene. 2009;28(Suppl 1):S24-S31.

2. Mok TS, Wu YL, Thongprasert S, et al. Gefitinib or carboplatinpaclitaxel in pulmonary adenocarcinoma. N Engl J Med. 2009;361(10): 947-957.

3. Murray S, Dahabreh IJ, Linardou H, Manoloukos M, Bafaloukos D, Kosmidis P. Somatic mutations of the tyrosine kinase domain of epidermal growth factor receptor and tyrosine kinase inhibitor response to TKIs in non-small-cell lung cancer: an analytical database. J Thorac Oncol. 2008;3(8):832-839.

4. Eisenhauer EA, Therasse P, Bogaerts J, et al. New response evaluation criteria in solid tumours: Revised RECIST guideline (version 1.1). Eur J Cancer. 2009;45(2):228-247.

5. Tseng YH, Tseng YC, Lin YH, et al. Epidermal growth factor receptor (EGFR)-tyrosine kinase inhibitor treatment and salvage chemotherapy in EGFR-mutated elderly pulmonary adenocarcinoma patients. Oncologist. 2015;20(7):758-766.

6. Tanaka T, Matsuoka M, Sutani A, et al. Frequency of and variables associated with the EGFR mutation and its subtypes. Int $J$ Cancer. 2010;126(3):651-655

7. Barlesi F, Mazieres J, Merlio JP, et al; Biomarkers France contributors Routine molecular profiling of patients with advanced non-small-cell lung cancer: results of a 1-year nationwide programme of the French Cooperative Thoracic Intergroup (IFCT). Lancet. 2016;387(10026):1415-1426.
8. Zhou C, Wu YL, Chen G, et al. Erlotinib versus chemotherapy as first-line treatment for patients with advanced EGFR mutation-positive non-small cell lung cancer (OPTIMAL, CTONG-0802): a multicentre, openlabel, randomised, phase 3 study. Lancet Oncol. 2011;12(8):735-742.

9. Douillard JY, Ostoros G, Cobo M, et al. First-line gefitinib in Caucasian EGFR mutation-positive NSCLC patients: a phase-IV, open-label, single-arm study. Br J Cancer. 2014;110(1):55-62.

10. Rosell R, Carcereny E, Gervais R, et al. Erlotinib versus standard chemotherapy as first-line treatment for European patients with advanced EGFR mutation-positive non-small-cell lung cancer (EURTAC): a multicentre, open-label, randomised phase 3 trial. Lancet Oncol. 2012;13(3):239-246.

11. Sequist LV, Yang JC, Yamamoto N, et al. Phase III study of afatinib or cisplatin plus pemetrexed in patients with metastatic lung adenocarcinoma with EGFR mutations. Clin Oncol. 2013;31(27):3327-3334.

12. $\mathrm{Wu} \mathrm{YL}, \mathrm{Zhou} \mathrm{C}, \mathrm{Hu} \mathrm{CP}$, et al. Afatinib versus cisplatin plus gemcitabine for first-line treatment of Asian patients with advanced non-small-cell lung cancer harbouring EGFR mutations (LUX-Lung 6): an open-label, randomised phase 3 trial. Lancet Oncol. 2014;15(2):213-222.

13. Socinski M, Villaruz L, Ross J. Understanding mechanisms of resistance in the epithelial growth factor receptor in non-small cell lung cancer and the role of biopsy at progression. Oncologist. 2017;22(1):3-11.

14. Howlader N, Noone AM, Krapcho M, et al, editors. SEER Cancer Statistics Review, 1975-2011. Based on November; 2013 SEER data submission, posted to the SEER website, April 2014. Bethesda, MD: National Cancer Institute. Available from: http://seer.cancer.gov/ csr/1975_2011/. Accessed November 1, 2015.

15. Van Damme V, Govaerts E, Nackaerts K, Dooms C, Wauters I, Vansteenkiste J. Clinical factors predictive of long-term survival in advanced non-small cell lung cancer. Lung Cancer. 2013;79(1):73-76.

16. Ozkaya S, Findik S, Dirican A, Atici AG. Long-term survival rates of patients with stage IIIB and IV non-small cell lung cancer treated with cisplatin plus vinorelbine or gemcitabine. Exp Ther Med. 2012;4(6): 1035-1038.

17. Lin JJ, Cardarella S, Lydon CA, et al. Five-year survival in EGFRmutant metastatic lung adenocarcinoma treated with EGFR-TKIs. J Thorac Oncol. 2016;11(4):556-565.

18. Inoue A, Kobayashi K, Maemondo M, et al; North-East Japan Study Group. Updated overall survival results from a randomized phase III trial comparing gefitinib with carboplatin-paclitaxel for chemo-naïve nonsmall cell lung cancer with sensitive EGFR gene mutations (NEJ002). Ann Oncol. 2013;24(1):54-59.

19. Yoshioka H, Mitsudomi T, Morita S, et al. Final overall survival results of WJTOG 3405, a randomized phase III trial comparing gefitinib (G) with cisplatin plus docetaxel (CD) as the first-line treatment for patients with non-small cell lung cancer harboring mutations of the epidermal growth factor receptor (EGFR). J Clin Oncol. 2014;32(Suppl):8117.

20. Lee CK, Brown C, Gralla RJ, et al. Impact of EGFR inhibitor in non-small cell lung cancer on progression-free and overall survival: a meta-analysis. J Natl Cancer Inst. 2013;105(9):595-605.

21. Yang JC, Wu YL, Schuler M, et al. Afatinib versus cisplatin-based chemotherapy for EGFR mutation positive lung adenocarcinoma (LUXLung 3 and LUX-Lung 6): analysis of overall survival data from two randomised, phase 3 trials. Lancet Oncol. 2015;16(2):141-151.

22. Shigematsu H, Gazdar AF. Somatic mutations of epidermal growth factor receptor signaling pathway in lung cancers. Int J Cancer. 2006; 118(2):257-262.

23. Shepherd FA, Rodrigues Pereira J, Ciuleanu T, et al. National Cancer Institute of Canada Clinical Trials Group. Erlotinib in previously treated non-small-cell lung cancer. $N$ Engl J Med. 2005;353(2):123-132.

24. Haaland B, Tan PS, de Castro G Jr, Lopes G. Meta-analysis of first-line therapies in advanced non-small-cell lung cancer harboring EGFRactivating mutations. J Thorac Oncol. 2014;9(6):805-811.

25. Takeda M, Nakagawa K. Toxicity profile of epidermal growth factor receptor tyrosine kinase inhibitors in patients with epidermal growth factor receptor gene mutation-positive lung cancer. Mol Clin Oncol. 2017;6(1):3-6. 
26. Schneider CP, Heigener D, Schott-von-Römer K, et al. Epidermal growth factor receptor-related tumor markers and clinical outcomes with erlotinib in non-small cell lung cancer: an analysis of patients from German centers in the TRUST study. J Thorac Oncol. 2008; 3(12):1446-1453.

27. Benedetti G, Latini L, Galetta D, Colucci G, Crinò L. Epidermal growth factor receptor exon 19 deletions predict complete regression of multiple intracranial metastases in two cases of non-small cell lung cancer treated with erlotinib. J Thorac Oncol. 2009;4(7): 936-937.

28. Yosuke T, Katsuhiro M, Masahide F, et al. Efficacy of increased-dose erlotinib for central nervous system metastases in non-small cell lung cancer patients with epidermal growth factor receptor mutation. Cancer Chemother Pharmacol. 2011;68(4):1089-1092.

29. von Pawel J, Wagner H, Duell T, Poellinger B. Erlotinib in patients with previously irradiated, recurrent brain metastases from non-small cell lung cancer: two case reports. Onkologie. 2008;31(3):123-126.

30. Riely GJ, Pao W, Pham D, et al. Clinical course of patients with nonsmall cell lung cancer and epidermal growth factor receptor exon 19 and exon 21 mutations treated with gefitinib or erlotinib. Clin Cancer Res. 2006;12(3 Pt 1):839-844.

31. Jackman DM, Yeap BY, Sequist LV, et al. Exon 19 deletion mutations of epidermal growth factor receptor are associated with prolonged survival in non-small cell lung cancer patients treated with gefitinib or erlotinib. Clin Cancer Res. 2006;12(13):3908-3914.

32. Weber B, Sorensen BS, Knap MM, Madsen HH, Nexo E, Meldgaard P. Complete pathologic response in lung tumors in two patients with metastatic non-small cell lung cancer treated with erlotinib. J Thorac Oncol. 2011;6(11):1946-1949.
33. Hesketh PJ, Chansky K, Wozniak AJ, et al. Southwest Oncology Group phase II trial (S0341) of erlotinib (OSI-774) in patients with advanced non-small cell lung cancer and a performance status of 2 . J Thorac Oncol. 2008;3(9):1026-1031.

34. Lin $\mathrm{CC}$, Yang $\mathrm{CH}$. Epidermal growth factor receptor tyrosine kinase inhibitors in elderly or poor performance status patients with advanced non-small cell lung cancer. Targ Oncol. 2009;4(1):37-44.

35. Ferte C, Besse B, Dansin E, et al. Durable responses to erlotinib despite KRAS mutations in two patients with metastatic lung adenocarcinoma. Ann Oncol. 2010;21(6):1385-1387.

36. Lara PN, Moon J, Hesketh J, et al. SWOG S0709: randomized phase II trial of erlotinib versus erlotinib plus carboplatin/paclitaxel in patients with advanced non-small cell lung cancer and impaired performance status as selected by a serum proteomics assay. J Thorac Oncol. 2016; 11(3):420-425

37. Yang J. A Randomized Controlled Trial of Erlotinib versus Gefitinib in Advanced Non-Small-Cell Lung Cancer Harboring EGFR Mutations (CTONG0901). Oral presentation at: WCLC 2015, 16th World Conference on Lung Cancer; September 8, 2015; Denver, CO, USA. Available from: https://library.iaslc.org/search-speaker?search_speaker=25092. Accessed August 22, 2017.

38. Goldberg SB, Oxnard GR, Digumarthy S, et al. Chemotherapy with erlotinib or chemotherapy alone in advanced non-small cell lung cancer with acquired resistance to EGFR tyrosine kinase inhibitors. Oncologist. 2013;18(11):1214-1220.
OncoTargets and Therapy

\section{Publish your work in this journal}

OncoTargets and Therapy is an international, peer-reviewed, open access journal focusing on the pathological basis of all cancers, potential targets for therapy and treatment protocols employed to improve the management of cancer patients. The journal also focuses on the impact of management programs and new therapeutic agents and protocols on

\section{Dovepress}

patient perspectives such as quality of life, adherence and satisfaction The manuscript management system is completely online and includes a very quick and fair peer-review system, which is all easy to use. Visit http://www.dovepress.com/testimonials.php to read real quotes from published authors. 